\title{
Achados Dermatoscópicos do Líquen Estriado - Relato de Caso
}

\author{
Carlos Gustavo Carneiro de Castro', Ana Carolina Serra Gomes da Silva Rodrigues², Bartira Beninca Sartori³, Bruna Barroso \\ Gonçalves ${ }^{3}$, Nathalia Diirr Travaglia4, Renato Moura Braga ${ }^{3}$, Glaura Tinoco Plata' \\ 'Especialista em Dermatologia pela Sociedade Brasileira de Dermatologia (SBD)/Specialist in Dermatology by SBD, Brasil \\ ${ }^{2}$ Graduada em medicina pela Universidade Estácio de Sá/Graduated in Medicine, by Estácio de Sá University, Rio de Janeiro, Brasil \\ ${ }^{3}$ Graduada em medicina pela Universidade Federal Fluminense (UFF)/Graduated in Medicine, by UFF, Rio de Janeiro, Brasil \\ ${ }^{4}$ Estudante de medicina da Universidade Federal Fluminense (UFF)/Medical Student of UFF, Rio de Janeiro, Brasil
}

RESUMO - Líquen estriado é uma erupção papulosa inflamatória que acomete principalmente crianças. Apresenta etiologia desconhecida e uma baixa prevalência. Surge menos frequentemente na idade adulta, e é caracterizado pelo aparecimento abrupto e assintomático de pápulas descamativas em disposição linear, por vezes seguindo as linhas de Blaschko, usualmente unilaterais e em extremidades. É importante considerar no diagnóstico diferencial outras dermatoses que acompanham as linhas de Blaschko, como por exemplo o líquen plano linear. Relata-se um caso atípico de líquen estriado com surgimento na idade adulta e localizado em flanco e região lombar à esquerda. Discutimos sobre a importância da dermatoscopia como ferramenta auxiliar no diagnóstico de líquen estriado.

PALAVRAS-CHAVE - Dermatoscopia; Erupções Liquenoides.

\section{Dermatoscopic Findings of Lichen Striatus - Case Report}

ABSTRACT - Lichen striatus is an inflammatory papular eruption that affects mainly children. It has an unknown etiology and a low prevalence. It arises in adulthood less frequently, and is characterized by abrupt onset of asymptomatic scaly papules in linear arrangement, sometimes following the Blaschko lines, usually unilateral and on the extremities. It is important to consider on the differential diagnosis other dermatoses that follow the Blaschko lines, such as linear lichen planus. We report an unusual case of lichen striatus with onset on the adulthood and located on left flank and on the left lumbar region. We discuss about the importance of dermatoscopy as an auxiliary tool on the diagnosis of lichen striatus

KEY-WORDS - Dermatoscopy; Lichenoid Eruptions.

\section{INTRODUÇÃO}

O líquen estriado é uma dermatose pouco prevalente, mais comum em crianças e adolescentes de 2 a 15 anos, principalmente do sexo feminino. ${ }^{4}$ Trata-se de uma doença inflamatória de etiologia ainda não esclarecida. Muitos autores, entretanto, a consideram como uma manifestação de mosaicismo, caracterizada pela presença de clones de células epiteliais alteradas geneticamente que, devido a um evento precipitante, são reconhecidas pelo sistema imune e podem induzir a pele afetada a gerar uma resposta inflamatória mediada por células T, em apresentação linear. ${ }^{4,6}$ Infecções, trauma, gravidez, drogas, atopia e fatores ambientais são descritos como prováveis precipitantes do líquen estriado, com a maioria dos casos ocorrendo durante a primavera e o verão. ${ }^{4,8}$

Clinicamente, esta dermatose é caracterizada por surgimento súbito de pápulas descamativas não pruriginosas, geralmente unilaterais que acometem principalmente as extremidades e tipicamente seguem as linhas de Blaschko. Raramente acomete tronco e face, mas pode ocorrer acometimento ungueal. ${ }^{8} \mathrm{Na}$ histopatologia observamos reação liquenóide com vacuolização da camada basal e inflamação de anexos. ${ }^{8}$
Correspondência: Dr. Renato Moura Braga

Rua Voluntários da Pátria 445, sala 702

Botafogo, Rio de Janeiro - RJ, Brasil, CEP 22270-000

E-mail: renato.moura.b@gmail.com
Recebido/Received

Outubro/October 2015

Aceite/Accepted

Dezembro/December 2015 


\section{Dermatoscopia}

O líquen estriado deve ser diferenciado de outras dermatoses que acompanham as linhas de Blaschko, dentre as quais podemos citar a psoríase linear e o líquen plano linear, com o qual pode haver sobreposição. ${ }^{4,8} \mathrm{O}$ tratamento baseia-se no uso de corticóide tópico ou inibidores de calcineurina ou imiquimod. ${ }^{9}$ Geralmente ocorre regressão espontânea entre 3 a 12 meses, com hipo ou hiperpigimentação. ${ }^{4}$

Relatamos a seguir um caso atípico de líquen estriado com surgimento na idade adulta e localização em tronco. Foi utilizado tratamento tópico com melhora importante das lesões e hipercromia residual.

\section{CASO CLÍNICO}

Paciente do sexo masculino com 32 anos de idade iniciou o quadro há aproximadamente 2 anos com surgimento progressivo de pápulas achatadas e hipercrômicas pruriginosas

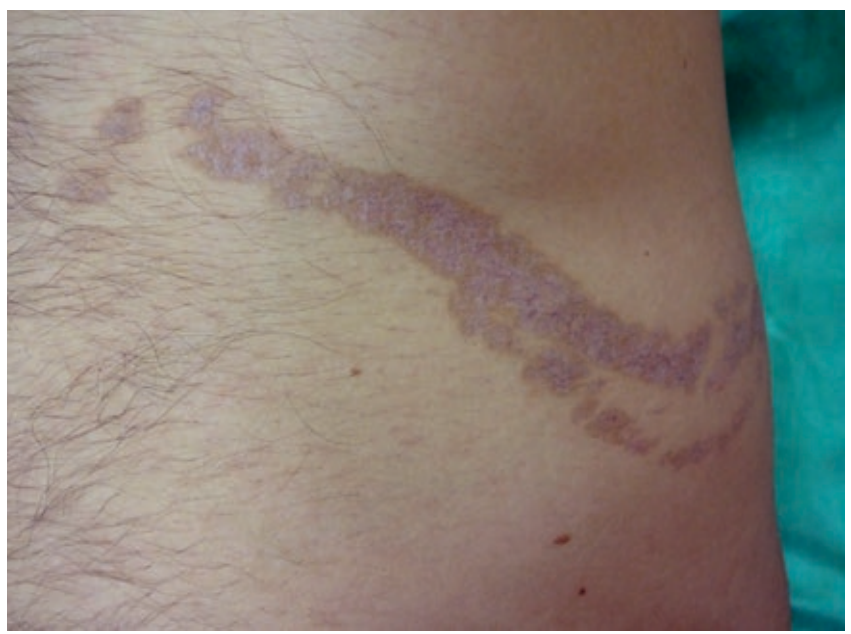

Figura 1 - Pápulas achatadas agrupadas hipercromicas seguindo as linhas de Blaschko.

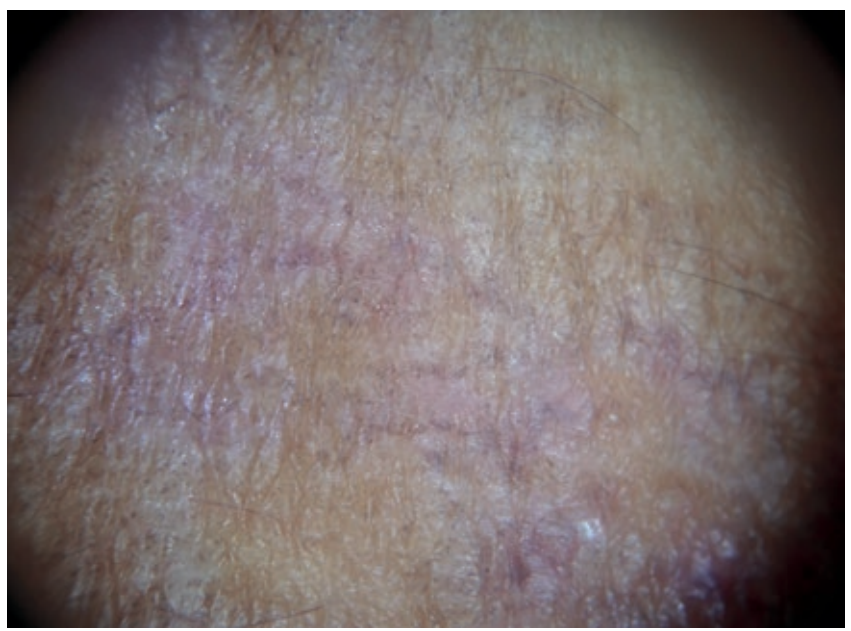

Figura 2 - Ao exame dermatoscópico: pontos violáceos entremeados por áreas esbranquiçadas.

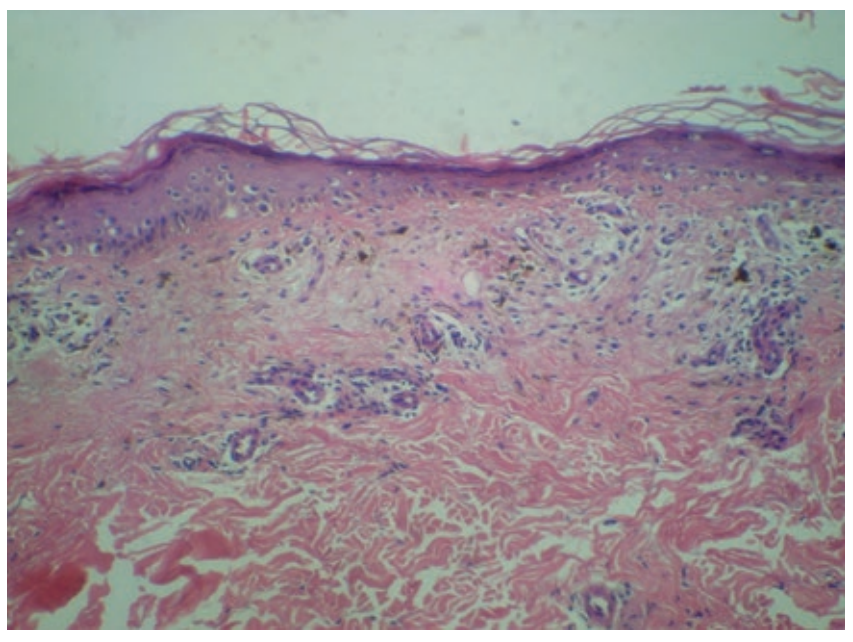

Figura 3 - Infiltrado inflamatório linfocitário na derme papilar perivascular e perianexial

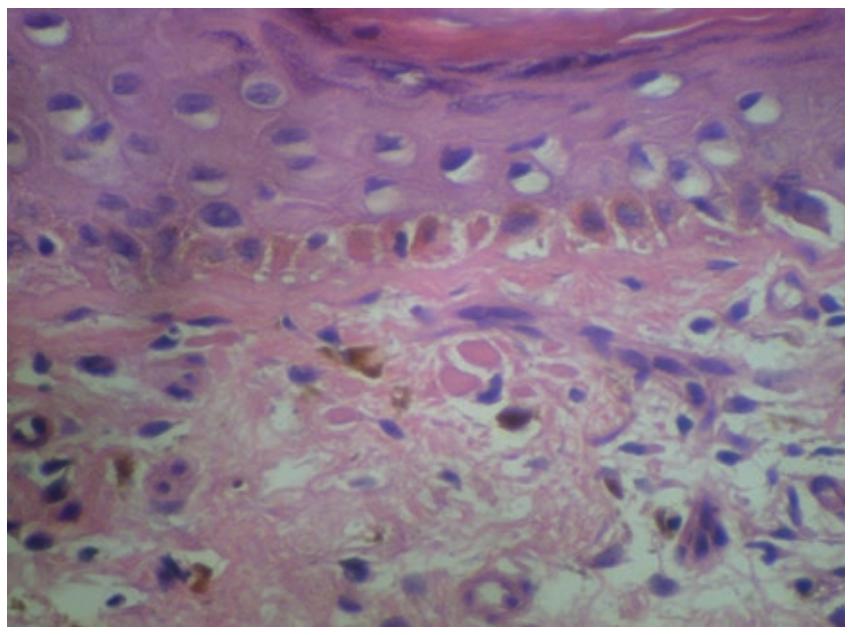

Figura 4 - Vacuolização da camada basal

em flanco esquerdo e e região lombar esquerda. Relata asma na adolescência. Nega história de líquen estriadona família. Nega doenças autoimunes na família.

Ao exame dermatológico foram observadas pápulas pequenas em torno de $2 \mathrm{~mm}$ de diâmetro, distribuídas no flanco esquerdo e a região lombar esquerda, seguindo as linhas de Bhlaschko. Na dermatoscopia, foram observados pontos violáceos entremeados por áreas esbranquiçadas. Foi realizada uma biópsia excisional em um pápula localizada no flanco esquerdo.

O exame histopatológico demonstrou epiderme com hiperceratose e vacualização da camada basal, infiltrado inflamatório linfocitário em faixa na derme papilar, perivascular e perianexial e, presença de melanófagos.

Foi iniciado tratamento com tacrolimus $0,1 \%$ pomada, duas vezes ao dia, durante 1 mês inicialmente. Relata que houve uma melhora importante das lesões após o tratamento, porém ocorrendo hipercromia residual. 


\section{DISCUSSÃO}

O líquen estriado é uma condição rara, auto-limitada e acomete principalmente crianças, em especial o sexo feminino. $^{7}$ Consiste em lesões papulosas pequenas, medindo de 2-4mm, superfície plana, levemente descamativas, podendo variar sua coloração entre o eritemato-violáceo ao hipocrômico. Geralmente se dispõem em conformação linear e são unilaterais, normalmente em extremidades, seguindo as linhas de Blaschko. Essas linhas são projeções do desenvolvimento celular normal na pele. Normalmente são invisíveis sob a pele normal mas são frequentemente afectadas nas dermatoses inflamatórias adquiridas. ${ }^{6}$ Aqui relatamos o caso de um paciente do sexo masculino, adulto, que desenvolveu um quadro de pápulas planas hipocrômicas em tronco(flanco esquerdo e região lombar esquerda). Essas lesões são descritas como assintomáticas em crianças, porém quando ocorrem em adultos pode ter prurido e requer tratamento sintomático, como no caso descrito ${ }^{3}$. Pode haver, também, mas com menos frequência, acometimento ungueal, apresentando-se com onicólise distal, estrias longitudinais e helconixe, ${ }^{4}$ achados esses que não estavam presentes no paciente em questão.

Múltiplos estudos mostram a associação do líquen estriado com atopia (asma, rinite alérgica ou dermatite atópica), chegando a $40 \%$. Isso é explicado pela desregulação da imunidade destes pacientes. Gravidez, trauma e drogas também são fatores de risco. ${ }^{1,2,9}$ Neste relato, o paciente possui história de atopia, porém nega história pessoal ou familiar de doenças auto-imunes.

O diagnóstico, em crianças, é eminentemente clínico, porém em adultos, pela sua baixa prevalência, é necessária confirmação histopatológica, especialmente se a lesão for encontrada fora das extremidades. ${ }^{1}$ A histopatologia mostra lesões infiltrado linfo-histiocitário e liquenóide, espongiose e inflamação perianexial superficial e profunda. Seu principal diagnóstico diferencial deve ser feito com o líquen plano linear. Este cursa com lesões violáceas e poligonais, estrias de Wickham, também seguem as linhas de Blaschko, e tem caráter pruriginoso. Na histopatologia apresenta infiltrado liquenóide na derme papilar. Outros diagnósticos diferenciais importantes são o lúpus eritematoso, psoríase, erupção liquenóide a droga, entre outros. ${ }^{6}$

A presença de pontos enegrecidos com aspecto salpicado e áreas esbranquiçadas na dermatocospia são compatíveis com líquen estriado. ${ }^{11}$ No caso relatado foram observados na dermatoscopia pontos violáceos e áreas esbranquiçadas na dermatoscopia.

Na maioria dos casos há involução espontânea da lesão entre um período de 3 a 12 meses, sendo possível, assim, uma conduta expectante. Quando não há resolução, o tratamento mais utilizado é com corticoterapia tópica. Também é relatada terapia com corticóide oral, inibidores de calcineurina tópicos e acitretina oral. ${ }^{5}$ Nosso paciente respondeu com regressão das lesões após 1 mês de tacrolimus tópico. Houve, entretanto, hipercromia residual, como já foi descrito na literatura. ${ }^{5}$
Os achados dermatoscópicos descritos neste relato podem ajudar a diferenciar o líquen estriado de outras dermatoses que seguem as linhas de Bhlaschko evidenciando a importância do dermatoscópio fazer parte da semiologia dermatológica.

Conflitos de interesse: Os autores declaram não possuir conflitos de interesse. Suporte financeiro: $O$ presente trabalho não foi suportado por nenhum subsídio ou bolsa. Confidencialidade dos Dados: Os autores declaram ter obtido consentimento escrito dos doentes para utilização dos seus dados clínicos e ter seguido os protocolos do seu centro de trabalho acerca da publicação dos dados de doentes.

Conflicts of interest: The authors have no conflicts of interest to declare. Financing Support: This work has not received any contribution, grant or scholarship. Privacy policy and informed consent: The authors declare that they have obtained informed consent from patients for the use of their medical data and followed the rules of the ethic committee of their center.

\section{REFERÊNCIAS}

1. Al Ratrout JT, Al Nazer N, Ansari NA.LichenStriatus in an Adult Female. Female, J Health Sci.2015; 5:14-6.

2. Brennand $\mathrm{S}$, Khan $\mathrm{S}$, Chong $\mathrm{AH}$. Lichen Striatus in a pregnant woman. Australas J Dermatol. 2005; 46:184-6.

3. Campanati A, Brandozzi G, Giangiacomi $M$, Simonetti $O$, Marconi B, Offidani AM. Lichen striatus in adults and pimecrolimus: open, off-label clinical study. Int J Dermatol. 2008;47:732.

4. Consuegra DG, Fabris MR, Vieira AG, Gama CM, Marquezini MA, Brotas AM. Líquen estriado no adulto com acometimento do aparelho ungueal. Rev Brasil Med. 2011. 2011 ; 13:20-1.

5. Errichetti E, Stinco G Trevisan G, Patrone P. Lichen striatus associated with psoriasis vulgaris treated with oral acitretin. Indian Dermatol Online J. 2014: 5: 501-503.

6. Fogagnolo L, Barreto JA, Soares CT, Marinho FC, Nassif PW. Líquen estriado no adulto. An Bras Dermatol. 2011; 86:142-5.

7. Hofer T. Lichen Striatus in Adults or "Adult Blaschkitis"? There is no need for a new naming. Dermatology. 2003; 207:89-92.

8. Kurokawa M, Kikuchi H, Ogata K, Setoyama M. Bilateral LichenStriatus. J Dermatol. 2004; 31:129-32.

9. Ramos C, Rodríguez S, Quijano E, Gutiérrez Z, Cortez-Franco F, Fuertes C, et al. Liquen estriado del adulto: experienciaenunservicio de Dermatología. Dermatol Peru. 2012; 22:16-20.

10. Zhang Y, McNutt NS. Lichen striatus. Histological, immunohistochemical, and ultrastructural study of 37 cases. J Cutan Pathol. 2001; 28:65-71.

11. Segura P, Romero M, Gonzalvo P, Garcia S, Iglesias J, Juanes J. Lichen striatus in na adult folowing trauma with central nail plate involvement and its dermoscopy features. Int J Dermatol. 2008, 47:324-5. 